\title{
MODULATION OF INTRA-EPITHELIAL EXPANSION OF HUMAN T24 BLADDER-CARCINOMA CELLS IN MURINE UROTHELIUM BY GROWTH FACTORS AND EXTRACELLULAR-MATRIX COMPONENTS
}

\author{
Johanna M.J. Rebel, Cornelia D.E.M. ThiJsSen, Marcel Vermey, Ellen C. ZWARThoff and Theodorus H. VAN Der Kwast \\ Department of Pathology, Erasmus University, P.O. Box 1738, 3000 DR Rotterdam, The Netherlands.
}

\begin{abstract}
The high recurrence rate of bladder cancer is probably due to an efficient repopulation of the bladder by residual transformed cells after resection of the tumour. However, the regenerating capacity of the normal urothelial cells is very high. To study the balance between regenerating normal urothelium and outgrowth of transformed urothelial cells, we recently developed an in vitro co-cultivation model. With this model system we studied the effects of growth factors and extracellular-matrix components on the intra-epithelial expansion of human T24 bladder-carcinoma cells in primary mouse-bladder explants. Exposure of the cultures to acidic fibroblast growth factor (aFGF) and laminin led to a dramatic increase in the number of invasive T24 cells into the primary urothelium. Epidermal growth factor (EGF) and collagen types I and IV counteracted the infiltration of individual T24 cells. EGF, aFGF, laminin and collagen types I and IV did not directly affect the migration and proliferation of T24 cells. Apparently, the efficacy of invasion of transformed urothelial cells into primary urothelium is not only dependent on the intrinsic characteristics of the transformed cells, but can be influenced to a considerable extent by exogenous components exerting their influence on the norma urothelium. The clinical relevance of this observation needs to be studied further.

0 1995 Wilev-Liss, Inc
\end{abstract}

Over $70 \%$ of human bladder carcinomas are superficial carcinomas and only $15 \%$ of them progress to invasive tumours after infiltration through the basement membrane (Levi et al., 1993). It is known that after local resection the frequency of tumour recurrence, in particular that of well-differentiated papillary transitional-cell carcinoma (TCC), is very high, and that these recurrent tumours may ultimately progress to invasive tumours (Heney et al., 1983; Raghavan et al., 1990). Carcinoma in situ of the flat urothelium is often found in association with invasive high-grade papillary and nonpapillary TCC. Originally it was thought that the recurrent bladder carcinomas represented de novo tumours arising from the dysplastic urothelium. However, it has recently been demonstrated that multiple, simultaneously occurring bladder carcinomas, including high-grade $\mathrm{TCC}$, in a single cystectomy specimen, as well as multiple high-grade tumour recurrences, are of monoclonal origin (Sidransky et al., 1992; Habuchi et al., 1993). These data imply that tumour recurrences in the bladder are often derived from a single progenitor cell and are most probably the consequence of intraluminal seeding or replacement of normal urothelium by neoplastic urothelial cells.

In a previous in vitro study, we showed that transformed urothelial cells can indeed replace primary urothelium (Rebel et al., 1993). It is not yet clear by what mechanisms (pre-)neoplastic urothelial cells can replace the normal urothelium. Several findings suggest that growth factors could be involved in the growth of TCC. Epidermal growth factor (EGF) is found in high concentrations in normal urine (Lau et al., 1988). On the other hand, an increased amount of acidic fibroblast growth factor (aFGF) and transforming growth factor $\alpha$ (TGF $\alpha$ ) was found in the urine of patients with bladder cancer, as compared with that of normal individuals. In addition, the expression of aFGF and basic fibroblast growth factor (bFGF) in TCC cells was higher than in normal urothelium (Kimball et al., 1984; Chodak et al., 1988; Ravery et al., 1992).
Intra-epithelial spread of transformed epithelial cells can be considered the consequence of cellular migration. Extracellularmatrix (ECM) components can promote cell spreading and the migration of different kinds of cells, e.g., during regeneration and wound healing (Nishida et al., 1990; Calof and Lander, 1991). These combined data prompted us to consider growth factors and ECM components as potentially important factors determining the level of intra-epithelial expansion of transformed epithelial cells.

In a previous study, we showed that organotypic cultures on transparent cyclopore membranes are ideally suited to the study of the expansion rate and degree of differentiation of the primary bladder culture under the influence of growth factors and ECM components (Rebel et al., 1994). In addition, we reported that the human T24 human bladder-carcinoma cell line expanded into the primary urothelial outgrowth (Rebel et al., 1993). We have now studied the influence of growth factors and ECM components on this process, and the results obtained show that exposure to aFGF and coating of the supportive membranes with laminin stimulate the invasion of T24 cells, while EGF treatment or coating with other ECM components counteracted intra-epithelial T24-cell invasion.

\section{MATERIAL AND METHODS}

Bladder explant cultures on transparent membranes

Urinary bladders were obtained from 6- to 8-week-old female $\mathrm{C} 3 \mathrm{H} / \mathrm{HE}$ mice. The mucosa was stripped from the underlying muscle layer. Half of the bladder mucosa was placed on a transparent membrane (Falcon cell-culture insert, Becton-Dickinson, Etten Leur, NL; cyclopore membrane with a diameter of $25 \mathrm{~mm}$; pore size $0.45 \mu \mathrm{m}$ ) with the lamina propria in contact with the membrane. The medium of the lower and upper compartment was changed every other day with standard medium, i.e., a 1:1 mixture of Dulbecco's modified Eagle's medium and HAM's F10 with $10 \%$ heatinactivated FCS and supplements as described (Rebel et al., 1993; De Boer et al., 1994).

When required, growth factors were added to the standard medium: aFGF, $20 \mathrm{ng} / \mathrm{ml}$, obtained from Boehringer Mannheim (Almere, NL) or EGF, $20 \mathrm{ng} / \mathrm{ml}$, obtained from Sigma (St. Louis, MO). Previous studies have indicated that these doses of aFGF or EGF induce enhanced expansion or proliferative activity, respectively, of the murine urothelium (Rebel et al., 1994). In other experiments, the transparent membranes were pre-coated with $25 \mu \mathrm{g} / \mathrm{ml}$ fibronectin (FN), laminin (Lam), collagen type I ( $\mathrm{Col}$ I) or with collagen type IV (Col IV) before the bladder explants were placed on the membrane as described (Rebel et al., 1994).

For the co-cultivation studies, 2 sheets of T24 cells of $5 \mathrm{~mm}^{2}$ were inoculated on opposite sides to the outgrowth (on day 4) of the bladder explant on day 4. The cell sheets were allowed to

${ }^{1}$ To whom correspondence and reprint requests should be sent. Fax: $31-10-4366660$

Received: September 9, 1994 and in revised form November 2, 1994. 
attach to the transparent membrane for $2 \mathrm{hr}$, then the upper compartment was refilled with standard medium.

\section{Immunohistochemistry}

The membranes were fixed with $70 \%$ ethanol for $24 \mathrm{hr}$. The membranes were pre-incubated with $10 \%$ normal goat serum for $15 \mathrm{~min}$ and then incubated with the primary antibody RCK 108 overnight at $4^{\circ} \mathrm{C}$. Subsequently, an indirect conjugatedperoxidase method was applied as described (Rebel et al., 1993). All membranes were counterstained with haematoxylin, dehydrated and mounted. The monoclonal antibody (MAb) RCK 108 (kindly provided by Dr. Ramaekers, University of Maastricht, The Netherlands) is specific for human cytokeratin 19, and does not cross-react with murine epithelia.

To evaluate the position of the immunostained T24 cells more accurately, strips of the immunostained transparent membranes covered with urothelial cells were dipped in $5 \%$ gelatin, dried overnight in air and embedded in paraffin. Cross-sections of $5 \mu \mathrm{m}$ were made perpendicular to the surface.

\section{Quantification of the distance of lateral invasion}

Quantification of the distance of lateral invasion of T24 cells in the primary bladder culture was done using an image analyzer (IBAS 2000 Zeiss Kontron, Oberkochen, Germany). The lateral invasion of T24 cells was visualized with a Hitachi CCTV camera and was analyzed with the supplied Kontron IBAS1 version 4.4 software program. The lateral infiltration distance of the T24 cells was the average of the most distant T24 cell found in the primary bladder culture at 30 random positions on the membrane.

Quantification of the number of invasive T24 cells was done by counting the number of T24 cells in 8 random fields of 0.15 $\mathrm{mm}^{2}$ at the edge of the urothelial outgrowth of the bladder explant. For statistical analysis we used Student's $t$-test.

\section{Proliferation and expansion assays}

To assess the effect of ECM components on proliferation of T24 cells, $1 \times 10^{3} \mathrm{~T} 24$ cells were seeded in 96-well dishes coated with $25 \mu \mathrm{g} / \mathrm{ml} \mathrm{Lam}, \mathrm{FN}$, Col I or Col IV. The effect of growth factors on the proliferation of T24 cells was investigated with non-coated dishes. On day $1,20 \mathrm{ng} / \mathrm{ml}$ aFGF or EGF were added. After 5 days of culture, $0.5 \mu \mathrm{Ci} / \mathrm{ml}{ }^{3} \mathrm{H}$ thymidine was added to each well, and the cells were incubated for another $16 \mathrm{hr}$. The cells were trypsinized and harvested. The ${ }^{3} \mathrm{H}$-thymidine incorporation was counted using a BetaPlate scintillator counter (LKB-Pharmacia, Woerden, The Netherlands) and expressed as cpm.

To assess the effects of ECM components and growth factors on the expansion of the T24 cells, a sheet of T24 cells of $5 \mathrm{~mm}^{2}$ was placed on a transparent membrane in the various conditions. The diameter of each T24 colony was measured every day. The measurements made during the first 10 days were then used to calculate the speed of cell expansion. After 14 days the T24 cells were harvested using trypsin, and the number of cells was counted.

A dose of $20 \mathrm{ng} / \mathrm{ml}$ aFGF was tested as chemo-attractant for T24, using a modified Boyden chamber assay with $8-\mu \mathrm{m}$ nuclepore filters (Keizer et al., in press). Incubation was for 4 hr at $37^{\circ} \mathrm{C}$ in the absence of FCS. The number of cells that migrated to the opposite side of the filter was counted.

\section{RESULTS}

\section{Effects of growth factors on the lateral invasion of T24 cells}

In explant cultures kept in standard medium containing $10 \%$ FCS, the urothelium immediately began to expand as a cohesive sheet of urothelial cells after their attachment to the cyclopore membrane. On day 4, when the urothelial outgrowth covered more than $8.5 \%$ of the membrane, sheets of T 24 cells were placed on the cyclopore membrane on each side of the outgrowth. On day 5 the standard medium was renewed, or replaced by standard medium containing aFGF or EGF. Between days 7 and 9, the T24 cells and the growing urothelium came into contact. After day 12 the surface area of the bladder outgrowth reached a plateau, since the T24 cells became continuous with the primary outgrowth. The cultures were terminated on day 24 and immunostained with RCK 108. In overview sections it was apparent that, in the control cultures (without growth factors added to the standard medium), small numbers of individual T24 cells were infiltrating the primary bladder culture (Fig. $1 a$ ). In contrast, in cultures treated with aFGF the T24 cells massively invaded the periphery of the primary urothelium, while in cultures treated with EGF much smaller numbers of T24 cells infiltrated the primary urothelium (Fig. $1 b, c$ ). The number and lateral distance of individual infiltrating T24 cells in the EGF-treated cultures were significantly smaller than those of the control or the aFGF-treated cultures $(p \leq 0.01)$ (Fig. $2 a, b)$. On crosssections, the T24 cells expanded by growing underneath the primary urothelium in the aFGF-treated and the control cultures. In the EGF-treated cultures the T24 cells were found on top of the, in this case, thickened rim of the primary urothelial outgrowth, whereas small numbers of T24 cells also expanded underneath the bladder urothelium (data not shown).

\section{Effects of extracellular-matrix components on the lateral invasion} of $T 24$ cells

The explants were placed on transparent membranes precoated with Lam, FN, Col IV or Col I. On day 1 the explants started to expand on the membrane and on day 4 sheets of T24 cells were again inoculated next to the outgrowth. Also in these cases, from day 12 on, further bladder outgrowth was inhibited by the T 24 cells. The cultures were terminated on day 24 and immunostained with RCK 108 . Invasion of T24 cells into the bladder outgrowth grown on Col IV, Col I and FN was observed only incidentally (Fig. 1e). The number and lateral distance of individual infiltrating T24 cells in the cultures grown on laminin were significantly greater than those of cultures grown on Col I, Col IV and FN (Figs. 1d, $3 a, b$ ). On cross-sections it was found that under all circumstances the T24 cells were infiltrating the urothelium outgrowth by expanding underneath the urothelium (data not shown).

Effects of growth factors and ECM components on migration and proliferation of $T 24$ cells

EGF added to standard medium containing $10 \%$ FCS did not influence the ${ }^{3} \mathrm{H}$-thymidine incorporation as compared with that of the control T24 cell cultures. In aFGF-treated cultures, the ${ }^{3} \mathrm{H}$-thymidine incorporation decreased as compared with that of the control cultures. Culturing of T24 cells on Lam, FN, Col I or Col IV substrate did not affect ${ }^{3} \mathrm{H}$-thymidine incorporation (data not shown). In the expansion assay on transparent membranes, a significant decrease in expansion of the T24 cells was found on the FN-coated membranes. In the other cases no significant changes in expansion of T24 cells was observed when compared with the $10 \%$ FCS control cultures (data not shown). In order to study a potential effect of aFGF on the migration of T24 cells, we used a modified Boyden-chamber assay. In the chambers with aFGF, $187.7 \pm 22.9$ cells were found on the opposite site of the filter, vs. $185.6 \pm 21.8$ cells in control experiments. We conclude that aFGF does not affect the migration of T24 cells.

\section{DISCUSSION}

Carcinoma in situ represents the most common form of intra-epithelial neoplasm of the bladder and may be considered as the intra-epithelial propagation of tumour cells originating from an adjacent bladder carcinoma (Koss et al., 1974; 

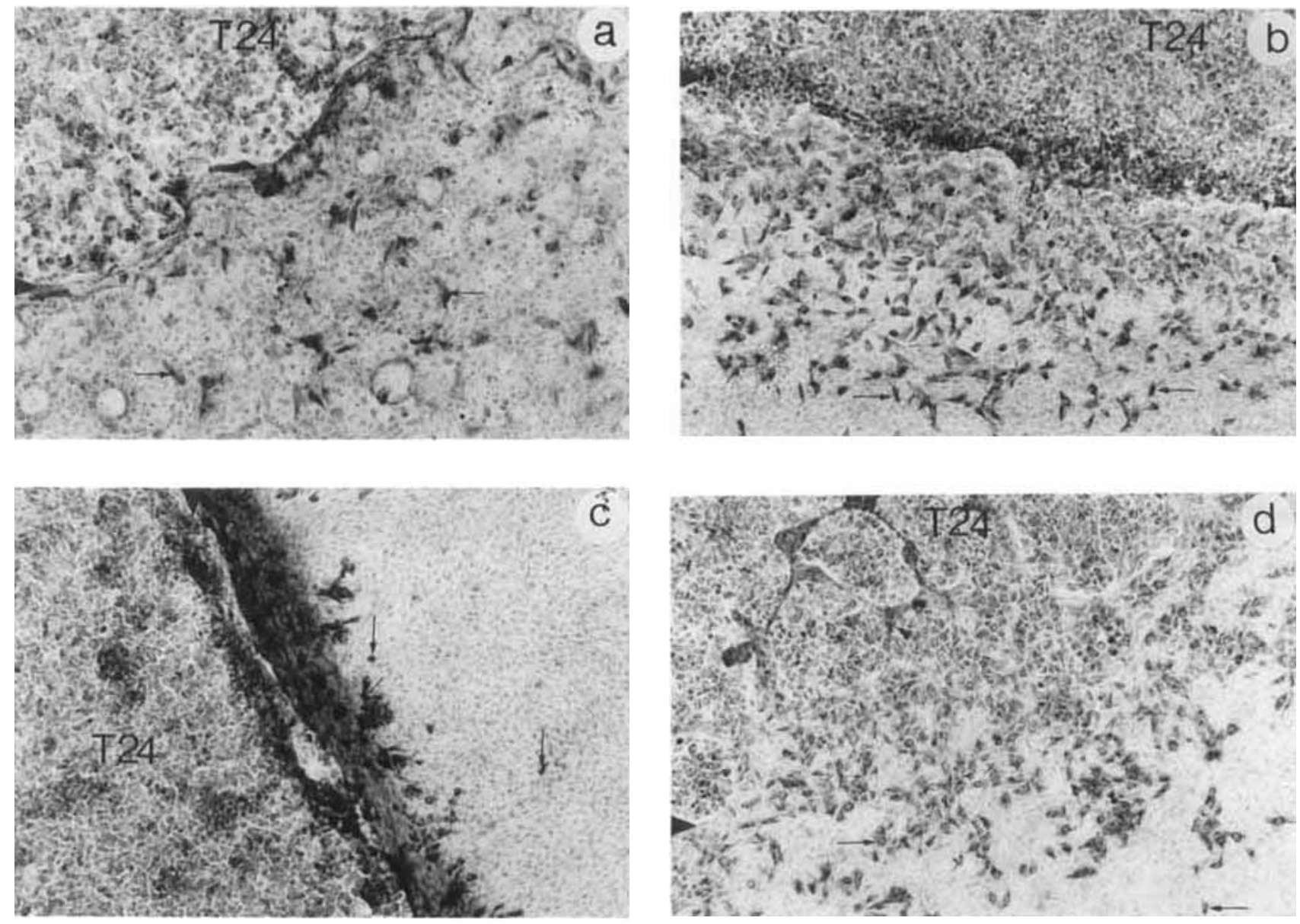

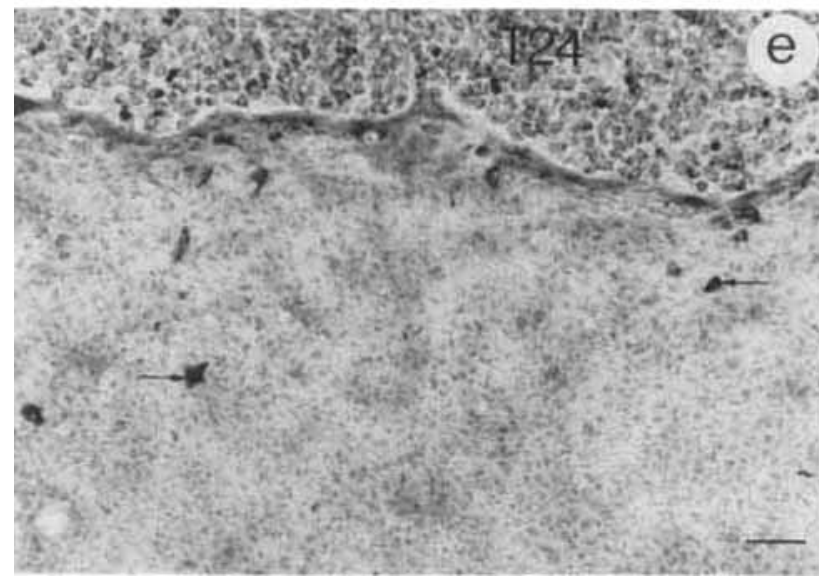

Mahadevia et al., 1986). The pagetoid type of infiltration is another form of intra-epithelial spread of bladder carcinoma, characterized by individual tumour cells infiltrating otherwise normal urothelium. Patients with the latter infiltration pattern have the same progression and survival rates as patients with a carcinomá in situ (Orozoco et al., 1993). The mechanisms underlying these 2 forms of intra-epithelial expansion have not been studied to a great extent, since in vitro models for intra-epithelial neoplasms are largely lacking.

Murine-bladder explants cultured on transparent cyclopore membranes expand rapidly, and the final cultures closely mimic the in vivo situation, even displaying maturation in umbrella cells. These cultures can easily be manipulated by
Figure 1 - Overview of co-cultivation of the primary urothelium and T24 cells $(\times 10)$. T24 cells are selectively stained with an antibody against cytokeratin 19. Nuclear counterstaining with haematoxylin. The marker $(\boldsymbol{A})$ indicates the border between T24 cells and the primary urothelial culture; arrows indicate individual infiltrating T24 cells in the primary urothelium. (a) Control culture; (b) culture treated with aFGF; (c) culture treated with EGF; (d) culture grown on laminin; $(e)$ culture grown on collagen type IV. Scale bar, $100 \mu \mathrm{m}$.

adding growth factors to the standard medium (containing $10 \%$ FCS) or by pre-coating the transparent membranes with extracellular-matrix components. Using this culture system, we here describe an in vitro model that yields quantitative and qualitative data on the intra-epithelial spread of human T24 cells into primary organotypic cultures of mouse urothelium. In the co-cultivation cultures, the T24 cells could be selectively immunostained with the species-specific monoclonal antibody (MAb) RCK 108, permitting their unequivocal identification (Rebel et al., 1993).

In control co-cultivation cultures, we found a pagetoid infiltration pattern of the T24 cells into the urothelium, below the primary urothelium. We presume that an interaction of the 

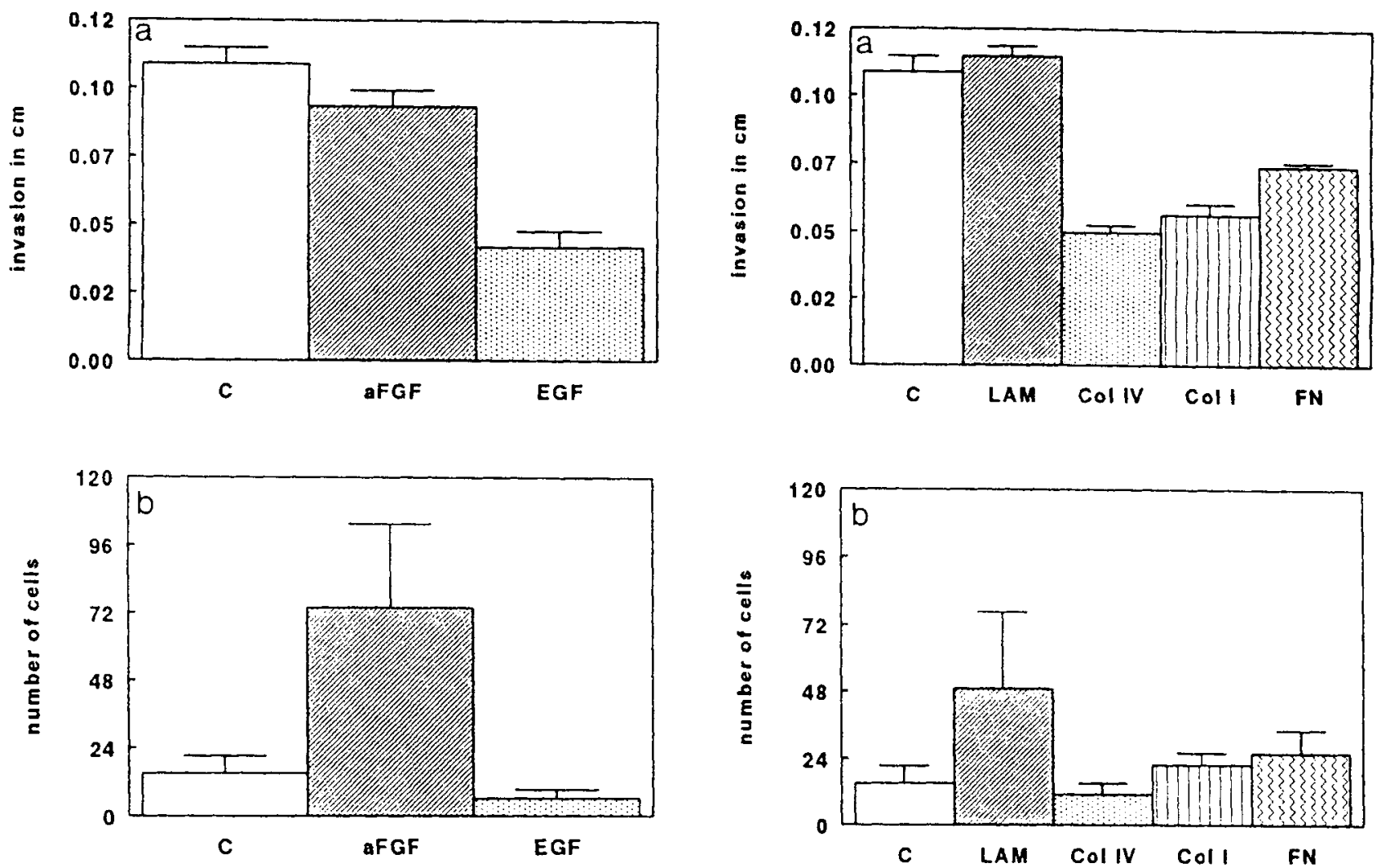

FIGURE 2 - Quantification of the lateral invasion of T24 cells in the explant cultures. The bars represent mean $\pm \mathrm{SE}$ of 3 independent experiments with 3 samples each. $c=$ control culture. (a) Distance of invasion of T24 cells in the primary culture in cm; (b) number of T24 cells infiltrating into the primary urothelium.

T24 cells with the supportive membrane is required for their intra-epithelial infiltration. Our observation that ECM coating of the supportive membranes specifically influences the extent and number of infiltrating T24 cells would reinforce the above hypothesis.

The observed growth factor and ECM-mediated variations in number and distance of infiltrating T24 cells into the urothelium could be due either directly, by action of these components on T24 cells or indirectly, via the primary urothelium. Migration of the T24 cells, however, was not affected by exposure to growth factors or ECM components, except for FN. Proliferation of T24 cells was also not affected by ECM or EGF exposure. On the other hand, these growth factors and ECM components strongly influence the expansion rate, and the level of differentiation and proliferative activity of the primary urothelial culture (Rebel et al., 1994). Therefore we conclude that the difference in number of and the distance covered by the infiltrating T24 cells in the primary urothelium must be ascribed to changes induced in the primary bladder culture, rather than a growth-factor- or ECM-componentmediated effect on the T24 cells. The smaller distance and lower number of infiltrating T24 cells in cultures grown on FN, however, could be due to direct inhibition of FN-mediated migration of T24 cells rather than a FN-mediated effect on the primary urothelium.

We have demonstrated that primary urothelial cultures exposed to EGF, Col I, Col IV and FN have an increased number of cell layers when compared with cultures grown on laminin (Rebel et al., 1994). In the aFGF-treated urothelium a decrease in nuclear density occurred in the periphery of the urothelium, when co-cultivated with T24 cells (Fig. 1b). Inter-

Figure 3 - Quantification of the lateral invasion of T24 cells in the explant cultures. The bars represent mean $\pm \mathrm{SE}$ of 3 independent experiments with 3 samples each. $c=$ control culture. (a) Distance of invasion of T24 cells in the primary culture in cm; (b) number of T24 cells infiltrating into the primary urothelium.

estingly, in the cultures exposed to EGF, Col I, Col IV and FN, with more than one cell layer in the periphery, a decrease in distance and number of infiltrating T24 cells was observed as compared with the urothelial cultures grown on laminin or treated with aFGF. Apparently, a reverse relation exists between cellular density of the primary urothelial culture and invasion of T24 cells.

Several studies have shown that growth factors can induce epithelial cells to produce ECM components. Fibronectin secretion by cornea epithelium and collagen-type-IV secretion by lung epithelium is enhanced by EGF (Federspiel et al., 1991; Schultz et al., 1992). Drago et al. (1991) showed enhanced laminin expression by neuro-epithelial cells after stimulation with bFGF. T24 cells express $\alpha 3, \alpha 5, \alpha 6, \beta 1$ and $\beta 4$ integrin sub-unit enabling them to attach to and interact with different ECM substrates (Coplen et al., 1991; Hogevorst et al., 1993). Thus, induction of urothelial ECM synthesis by aFGF could also be a possible underlying mechanism by which T24 cells are permitted to adhere and migrate underneath the primary urothelial outgrowth.

In summary, the balance of transformed urothelial cells and (regenerating) urothelium is dependent not only on direct effects of growth factors and extracellular matrix components on transformed cells but, as these data imply, also on factors directly affecting the primary urothelium.

\section{ACKNOWLEDGEMENTS}

This study was supported by the Dutch Cancer Foundation (IKR 90-17). We thank Miss S. Erkens for technical assistance and Mr. F.L. van der Panne for photography. 


\section{REFERENCES}

CALOF, A.L. and LANDER, A.D., Relationship between neuronal migration and cell-substratum adhesion: laminin and merosin promote olfactory neuronal migration but are anti-adhesive. J. Cell Biol., 115 , $779-794$ (1991).

Chodak, G.W., Hospelhorn, V., Judge, S.M., Mayforth, R. KOEPPEN, H. and SASSE, J., Increased levels of fibroblast growth-factorlike activity in urine from patients with bladder or kidney cancer Cancer Res., 48, 2083-2088 (1988).

Coplen, D.E., Brown, E.J., McGarr, J. and Ratliff, T.L., Characterization of fibronectin attachment by a human transitional-cell carcinoma line T24. J. Urol., 145, 1312-1315 (1991).

De Boer, W.I., Rerel, J.M.J., Vermey, M., Thussen, C.D.E.M. and VAN DER KWAST. T.H., Multiparameter analysis of primary cultures grown on cyclopore membranes. J. Histochem. Cytochem., 42, 277-282 (1994).

Drago, J., Nurcombe, V., Pearse, M.J., Murphy, M. and BartletT, P.F., Basic fibroblast growth factor up-regulates steady-state levels of laminin B1 and B2 chain mRNA in cultured neuro-epithelial cells. Exp. Cell Res., 196, 246-254 (1991).

Federspiel, S.J., DiMari, S.J., Howe, A.M., Guerry-Force, M.L and Haral.SON, M.A., Extracellular-matrix biosynthesis by cultured fetal-rat-lung epithelial cells. Lab. Invest., 64, 463-473 (1991)

Habuchi, T., Takahasi, R., Yamada, H., Kakehi, Y., Sugiyama, T. and YoshiDA, O., Metachronous multifocal development of urothelial cancers by intraluminal seeding. Lancet, 342, 1087-1088 (1993).

Heney. N.M., Ahmed, S., Flanagan, M.J., Frable, W., Corder, M.P., HafermanN, M.D. and Hawkins, I.R., Superficial bladder cancer: progression and recurrence. $J$. Urol., 130, $1083-1086$ (1983).

Hogevorst, F., Admiraal, L.G., Niessen, C., Kuikman, I., Janssen, H., DAAMS, H. and SONNENBERG, A., Biochemical characterization and tissue distribution of the $a$ and $b$ variants of the integrin $\alpha 6$ sub-unit. J. Cell Biol., 121, 179-191 (1993).

Keizer, D.M., Erkens, S., Romijn, J.C. and Schroeder, F.H. Production of motility factors, migratory response and actin filament organization of rat Dunning prostate-carcinoma cells with different metastatic potential. Clin. exp. Metast. (In press).

Kimball, E.S., Bohn, W.H., Cockley, K.D., Warren, T.C. and SHERWIN, S.A., Distinct high-performance liquid-chromatography pattern of transforming-growth-factor activity in urine of cancer patients as compared with that of normal individuals. Cancer Res., 44, 36163619 (1984)
Koss, L.G., Tiamson, E.M. and Robbins, M.A., Mapping cancerous and pre-cancerous bladder changes: a study of the urothelium in ten surgically removed bladders. J. Amer. med. Ass., 227, 281-286 (1974).

LAU, J.L.T., FOwlER, J.E. and GOSH, L., Epidermal growth factor in the normal and neoplastic kidney and bladder. J. Urol., 139, 170-175 (1988).

Levi, F., La Vecchia, C., Randimbison, L. and Franceschi, S., Incidence of infiltrating cancer following superficial bladder carcinoma. Int. J. Cancer, 55, 419-421 (1993).

Mahadevia, P.S., Koss, L.G. and Tar, I.J., Prostatic involvement in bladder cancer: prostate mapping in 20 cystoprostatectomy specimens. Cancer, 58, 2096-2102 (1986).

Nishida, T., Nakamura, M.. Mishima, H. and Otori, T,, Differential modes of action of fibronectin and epidermal growth factor on rabbit corneal epithelial migration.J. cell. Physiol. 145, 549-554 (1990).

OROZOCO, R.E., VAN DER ZWAAG, R. and MURPHY, W.M., The pagetoid variant of urothelial carcinoma in situ. Hum. Pathol, 24, 1199-1202 (1993).

Raghavan, D., Shipley, W.U., Garnick, M.B., Russell, P.J. and RICHIE, J.P., Biology and management of bladder cancer. New Eingl. J. Med., 322, 1129-1138 (1990).

Ravery, V., Jouanneau, J., Gil Diez, S., Abbou, C.C., Caruelle, J.P., BARRITAULT, D. and ChOPIN, D.K., Immunohistochemical detection of acidic fibroblast growth factor in bladder transitional-cell carcinoma. Urol. Res., 20, 211-214 (1992).

Rebel, J.M.J., DE BOER, W.I., ThIJSSEN, C.D.E.M., VERMEy, M., ZWARTHOFF, E.C. and VAN DER KWAST, T.H., An in vitro model of intra-epithelial expansion of transformed cells. Int. J. Cancer, 54, 846-850 (1993).

RebEl, J.M.J.. DE BoER, W.I., ThiJSSEN, C.D.E.M., VERMEy, M., ZWARTHOFF, E.C. and VAN DER KwaSt, T.H., An in vitro model of urothelial regeneration: effects of growth factors and extracellular matrix proteins. J. Pathol., 173, 283-291 (1994).

Schultz, G., Chegini, N., Grant, M., Khaw, P. and MacKay, S., Effects of growth factors on corneal wound healing. Acta Ophthalmol, 70, 60-66 (1992).

Sidransky, D., Frost, P., Von Eschenbach, A., Oyasu, B., PreisINGER, A.C. and VOGELSTEIN, B., Clonal origin of bladder cancer. New Engl. J. Med., 326, 737-740 (1992). 